should be undertaken with an awareness of these aspects and in a way that provides space to experiment with them. Some of the constellations outlined in this anthology suggest that this exercise requires a certain sensitivity for both the relational and epistemic potential of media. This becomes evident, as well, in the article by Enzo Camacho and Amy Lien, who visually reorganize stories around a mural of Christ in a church of a sugar refinery on the Philippine island of Negros. In that way, they create a narration that is more than a mere effect of existing semantic and linguistic orders. The practice of Camacho and Lien is just one example of how in arts and design there is often more courage to project new modes of relating. The recognition of this potential may be another reason for the elliptical character of the question How to relate ...? - which also alludes to a moment of speechlessness and the suspension of theoretical discourse. The ellipsis may be a first concession to this entanglement of agents of knowledge that we are part of and from within which we try to speak and write. The ellipsis leaves blank who and what are to be related or relate. It calls to be completed in a situated and precise manner - or for us to pose the question How to relate ...? also with nondiscursive media in order to instigate different conditions and relations.

\title{
Mirjam Schaub \\ Offen und entschieden: die idiosynkratische Radikalität der Kunst
}

\section{Einleitung}

Der Kunstdiskurs, der seine Werke umschmeichelt, sie in schöne Worte kleidet oder gelehrt und manchmal selbstverliebt um ihre mannigfaltigen Bedeutungen kreist, verkennt - so die These -, dass Kunstwerke selbst erstaunlich entschieden sind. Deshalb treffen Kunstwerke (und nicht erst die modernen!) ihre Betrachter_innen mitunter mit einer Erkenntnis, oft hart und mitten ins Gesicht, wie eine Beleidigung von unerwarteter Seite.

Kein Werk, so die zugrunde liegende Überzeugung, ist beliebig, sondern im Gegenteil auf idiosynkratische Weise entschieden. Diese Entschiedenheit folgt durchaus tautologisch aus der endlichen Gesamtheit künstlerischer Entscheidungen. Sie bestimmt sich aus den Eigenschaften der gewählten Materialien, Genres und Mischformen; verdankt sich dem Grad des 
handwerklichen (Un-)Vermögens; formt sich anhand der zugelassenen oder unvermeidlichen produktiven Zufälle, die Werkentstehung, Ausstellung und Rezeption begleiten; konturiert sich entlang der Reibungsflächen mit Konvention, Diskurs und Kunstraum. All diese Faktoren tragen zur Entschiedenheit eines Werkes bei, bilden seine endliche Figuration und Gestalt, was auch die besondere Performanz in einem Ausstellungskontext oder darüber hinaus (in einem Happening z. B.) einschließt.

Die endliche Figuration des Kunstwerks wird üblicherweise als seine ,Geschlossenheit ${ }^{c}$ bezeichnet. ${ }^{1}$ Hier möchte ich deren unterschätzte epistemische Wirkungen ausloten. Um diese zu akzentuieren, spreche ich von der,Entschiedenheit' der Kunstwerke. Wie aber geht diese mit der nicht bloß interpretativen, sondern strukturellen ,Offenheit' von Kunstwerken zusammen, wie sie Umberto Eco - weithin anerkannt - zumindest für moderne Werke unterstreicht? Stimmen beide Beobachtungen überhaupt oder beruhen sie auf einem Missverständnis?

Das Nachdenken über die Beziehungsweisen eines Kunstwerks, seine Verfransung mit Konvention und Diskurs, Wahrnehmen und Denken kann hier womöglich Abhilfe schaffen. $\mathrm{Zu}$ diesem Zweck möchte ich Eco mit Niklas Luhmann und Gilles Deleuze gegenlesen und anhand einer Arbeit von Santiago Sierra sowie einer Performance von Linda Montano und Tehching Hsieh die Entschiedenheit von Kunstwerken zeigen.

\section{Der Mythos vom ,offenen Kunstwerk}

Umberto Ecos Opera Aperta von 1962 ist ein Klassiker geworden, ein Schlüsseltext zum Verständnis moderner Kunstwerke. ${ }^{2}$ Offenheit bahnt sich für Eco bereits im Zeitalter des Barock ihren Weg in die Moderne, da die durch Illusionsmalerei dramatisch in Szene gesetzte Architektur die Betrachter_innen dazu zwinge, beständig den Standort zu wechseln, „um das Werk unter immer neuen Aspekten zu sehen, so als ob es in beständiger Umwandlung begriffen wäre".3 Der ideale Blickpunkt werde so immer wieder von Neuem gesucht, gefunden und wieder verworfen. Die ,sprechende ${ }^{6}$ Architektur des Barock mit seinen „plastischen Massen“ verlange dem Menschen erstmals ein "schöpferisch-erfinderisches Verhalten" ab, indem sie auf die Stimulanz der Einbildungskraft abziele und die Betrachter_innen so aus der „Norm des Kanonischen" befreie. ${ }^{4}$ Erst die eigene imaginatio, griechisch phantasía, vollende das barocke Kunstwerk. Offenheit meint für Eco seither vor allem eines: unvollendet zu sein, solange die Betrachter_innen der Kunst nicht selbst ,im Bild' sind und ,in den Blick' genommen werden.

1 Doch ist darunter mehr als „die ursprünglich vom Künstler imaginierte Form“ zu verstehen, siehe Eco, Umberto: Das offene Kunstwerk, übers. von Günter Memmert, Frankfurt a. M. 1977 [zuerst Mailand 1962, Opera Aperta], S. 29.

2 Vgl. Rebentisch, Juliane: Theorien der Gegenwartskunst zur Einführung, Frankfurt a. M., 2013, S. 31f.

3 Eco 1977, S. 35.

4 Ebd. 
Offen kann jedoch auch heißen: nicht nur unvollendet $\mathrm{zu}$ sein, sondern auch unfertig, unbestimmt, ungewiss, unentscheidbar, vielfältig interpretierbar. Tatsächlich reizt Eco das gesamte Bedeutungsspektrum des Offenen aus, wenn er das Phänomen für die Moderne zwischen struktureller und interpretativer Unbestimmtheit durchdekliniert. Er beginnt mit dem kleinsten gemeinsamen Nenner, mit dem Phänomen der Mehrdeutigkeit: „Das [moderne] Kunstwerk gilt als eine grundsätzlich mehrdeutige Botschaft, als Mehrheit von Signifikaten (Bedeutungen), die in einem einzigen Signifikanten (Bedeutungsträger) enthalten sind. "5 Der Signifikant ist einer, die Signifikate sind viele. Vor dem Hintergrund informationstheoretischer Überlegungen ersetzt Eco diese Mehrdeutigkeit jedoch nur wenige Seiten später durch eine „fundamentale[ ] Ambiguität der künstlerischen Botschaft", die „eine Konstante jedes Werkes aus jeder Zeit" darstelle. ${ }^{6}$

Damit baut Eco die unterstellte Offenheit des Kunstwerks als sprichwörtlichen Beziehungsreichtum in kategorialer Weise aus. So sieht er mit dem Symbolismus eines Verlaine oder Mallarmé eine regelrechte „Poetik des Andeutens" am Werk, die sich zu einem „Ausdruck des Unbestimmten“" auswachse, ${ }^{7}$ welche die emphatische Positionierung der Lesenden verlange. Was im Barock als unvollendet erscheint, weitet sich in der Musik des 20. Jahrhunderts, etwa bei Luciano Berio und Karlheinz Stockhausen, zum Eindruck der Unfertigkeit aus. Doch dahinter steckt etwas Anderes: Ecos Buch endet mit einer Hommage an James Joyce' Ulysses und Finnegans Wake. Es sind avantgardistische Poetiken wie diese, anhand derer Eco ableitet, Offenheit werde bewusst, d.h. strategisch, sowie regelrecht mit künstlerischen Mitteln als „ein vor anderen zu realisierender Wert" gesetzt. ${ }^{8}$

Im Vorwort zur zweiten Auflage von Opera Aperta setzt sich Eco 1977 sodann mit seinen Kritiker_innen auseinander. Er sieht sich zu einer Reihe von $\mathrm{Zu}$ spitzungen veranlasst, die für unseren Argumentationszusammenhang deshalb von Interesse sind, weil sie Offenheit als Möglichkeitsbedingung von Entschiedenheit auffassen. Eco rudert dabei argumentativ gleichzeitig vor und zurück: zurück, weil er die konkrete Anwendbarkeit seines Offenheitsbegriffs in Zweifel zieht; vor, weil er dessen konzeptuelle Berechtigung vehement verteidigt. Wie geht das?

Das ,offene Kunstwerk' sei weniger als eine ,objektive Struktur' der Werke selbst zu verstehen denn als eine „Rezeptionsbeziehung“9, d.h. als eine $B e$ ziehungsweise, die sich gerade dem stummen Dialog mit den Betrachter_innen verdanke. Offenheit meine weniger „eine kritische Kategorie“ denn ein „hypothetisches Modell, das zwar anhand zahlreicher konkreter Analysen ausgearbeitet wurde, aber eben doch nur dazu dient, mit einer bequemen Formel

5 Ebd., S. 8, Herv. d. Verf.

6 Ebd., S. 11.

7 Ebd., S. 37.

8 Ebd., S. 8.

9 Ebd., S. 15. 
eine Richtung der modernen Kunst zu bezeichnen“. Es handele sich bei dieser Offenheit - wie Eco nun nicht ohne leise Koketterie behauptet - um eine "Abstraktion". ${ }^{10}$ Ziel sei es gewesen, das Kunstwerk strukturell vergleichbar $\mathrm{zu}$ machen, unter anderem mit den experimentellen und hypothesengeleiteten Verfahren der Naturwissenschaftler_innen. In der Konkretion jedoch zeigten sich die Grenzen der Übertragbarkeit.

Ecos ,offenes Kunstwerk funktioniert als Modell deutlich besser für allografische, d.h. an performative Praktiken gebundene, aufführungsbasierte Künste wie Musik, Theater, Tanz, Performance-Kunst usf., als für autografische, d.h. vom Original abhängige und damit fälschungsempfindliche Künste wie Malerei und Skulptur. Eco ist sich dieser Problematik durchaus schmerzlich bewusst:

Und einigen Malern oder Romanschreibern, die uns nach der Lektüre dieses Buches ihre Werke mit der Frage vorlegten, ob es "offene Kunstwerke“ seien, mussten wir in scheinbar polemischer Starrheit antworten, dass wir „offene Kunstwerke“ noch nie gesehen hätten und es in Wirklichkeit wohl gar keine gebe. ${ }^{11}$

Dabei spitzt er das zugrunde liegende Problem mit Blick auf die Literatur weiter $\mathrm{zu}$ :

Es hat manche vor den Kopf gestoßen, daß das Arbeitsmodell des offenen Kunstwerkes sowohl auf ein informelles Bild wie auf ein Drama von Brecht Anwendung finden sollte. [...] Wenn in diesem Buch ein Modell des offenen Kunstwerkes weniger an Werken des Typus Brecht erarbeitet wird als an solchen, bei denen das formale Streben nach selbstzweckhaften Strukturen ausgesprochener und entschiedener ist, so rührt das daher, daß in diesen Werken das Modell leichter exemplifizierbar schien. ${ }^{12}$

Für unsere Frage der in die Offenheit eingesenkten Entschiedenheit sind jedoch ausgerechnet Werke „des Typus Brecht“ von Interesse. Denn Brechts Stücke sind, nach Ecos eigenem Bekunden, immer noch ein ziemlich isoliertes Beispiel für ein offenes Kunstwerk [...], das auf einen konkreten ideologischen Aufruf hinauswill; oder besser, das einzige deutliche Beispiel eines in die Form eines offenen Kunstwerkes gebrachten ideologischen Aufrufs. 13

10 Ebd., S. 12.

11 Ebd., S. 11.

12 Ebd., S. 13f., Herv. d. Verf.

13 Ebd., S. 14. 
Für Eco geht die dogmatische Entschiedenheit, der politische Impetus eines Brecht damit nicht umstandslos in seiner eigenen Theorie der Bedeutungsoffenheit auf. Er versteht gut, dass Brechts Werke nicht wirken, weil sie auf Offenheit abzielen. Im Gegenteil, sie wirken, weil sie radikal andere Interessen verfolgen.

Was Eco 1962 als „isoliertes Beispiel“ integriert, ist in meiner Lesart knapp 60 Jahre später eher zum Regelfall der modernen Kunst geworden. Wir haben es heute, gerade in der Installations- und in der Performance-Kunst und besonders auch im sogenannten ,Artivism (Peter Weibel), mit einem völlig anderen Typus von Kunstwerk zu tun. An dem - scheinbar - exzentrischen Beispiel Brechts lässt sich meines Erachtens präzise bestimmen, welche Wirkungen der strukturellen Rahmung durch eine Konzeption offener Kunstwerke Eco nicht voraussah.

Ich möchte im Unterschied zu Eco behaupten, dass gerade die strukturelle und nicht die interpretative Offenheit von Kunstwerken Raum gibt für die besondere Entschiedenheit, für die besonderen Formen künstlerischer Radikalität. Diese Radikalität, so die These, wird just dadurch akzeptabel, weil ihr ein institutioneller Spielraum an die Seite gestellt wird. Kunst ist ein sozial akzeptierter Schutzraum, ähnlich wie die Kindheit, in dem nichts allein im Modus des ,so ist es, sondern immer auch im Modus des Optativs, im Modus des ,so könnte es (auch) sein', ,das könnte es (auch) werden' existiert.

Aufgrund dieser künstlerischen, ja theatralischen Rahmung, die kein Verfremdungseffekt der Welt zu neutralisieren vermag, wirkt bis heute jedes Brecht-Stück anders und dabei politischer - oder wie Eco sagt: ausgesprochener und entschiedener - als der Besuch einer politischen Kundgebung. Es ist ausgerechnet die vergleichsweise harmlose Rahmung der Kunst, ihr augenzwinkerndes ,Als-ob', in dessen Schutz inhaltliche Härte und auch politische Entschiedenheit auf eine erträgliche und annehmbare Weise zur Geltung kommen und Gehör finden kann. Der offenkundige Clash aus permissiver Form und explosivem Inhalt erleichtert - so die These -, dass auch das Einseitige, Eindimensionale, Entschiedene darin akzeptabel bleibt. Es bleibt akzeptabel, solange eine sie rahmende Offenheit - struktureller, institutioneller, aber eben nicht primär interpretativer Natur - ausgleichend und einladend dahintersteht. Polemisch gesagt: Die Offenheit ist dann der Köder, in deren Schutz etwas passiert, was überhaupt nicht offen, sondern im Gegenteil höchst entschieden ist.

Warum verkennt oder ignoriert Eco diese reale und sehr greifbare Wirkung der von ihm entdeckten strukturellen Offenheit des Kunstwerks qua permissivem Kunstraum? Ich habe zwei Antworten auf diese Frage: erstens, weil die interpretative Seite der Offenheit am Beispiel der modernen Literatur die argumentative Oberhand über ihre strukturellen und institutionellen Gegenstücke gewinnt. Zweitens, weil Eco letztlich den Gedanken eines mimetischen Reflexes verfolgt, um seine Rede von der Offenheit against all odds aus einer Zeitdiagnose heraus zu motivieren. 
Immer wieder kommt Eco auf die frühen 1960er Jahre zu sprechen, in denen sich Europa von zwei Weltkriegen zu erholen beginnt, sowie auf die besonderen ,Ideale dieser Zeit nach der geschichtlichen Katastrophe. Die analytischen Begriffe, mit denen er die 1960er Jahre - auch aus einer italienischen Perspektive heraus - charakterisiert, lauten: Unbestimmtheit, Aleatorik, Unordnung. Die Offenheit der modernen Kunst ist für Eco damit letztlich eine treffliche Spiegelung, ein auf Symmetrie und ,Augenhöhe bedachter Versuch, diese neue Unübersichtlichkeit wieder in den Diskurs, auch den wissenschaftlichen, einzubetten. Denn Offenheit entsteht für Eco durch die Bestimmung jener Grenzen, „innerhalb derer ein Kunstwerk die größte Mehrdeutigkeit verwirklichen und von dem aktiven Eingriff des Konsumenten abhängen kann, ohne damit aufzuhören, Kunstwerk zu sein". ${ }^{14}$ Die Offenheit des modernen Kunstwerks wird damit als mimetischer, affirmativ-subversiver Reflex aufgefasst; als Anpassungsleistung an eine sich ambig und mehrdeutig zeigende, von Zufällen beherrschte Moderne.

Eco reagiert damit, wie er selbst zugibt, zeitdiagnostisch auf die neue ,Unübersichtlichkeit" (er selbst spricht lieber von „Unordnung“). Auch im Vorwort zur Neuauflage von 1977 kommt er noch einmal auf diese - offenbar keineswegs veraltete - Ausgangsdiagnose zurück, was ihre Wichtigkeit für seine Argumentation unterstreicht. Eco interessiert daran die „fruchtbar[e] Unordnung, deren Positivität die moderne Kultur uns gezeigt hat" und die sich u.a. ,in den Hypothesen der Unbestimmtheit, der statischen Wahrscheinlichkeit, den provisorischen und variablen Erklärungsmodellen" aufgelöst habe. Die Kunst habe „nichts weiter getan, als diese Situation zu akzeptieren und ihrer Berufung entsprechend - zu versuchen, ihr Form zu geben". ${ }^{15}$

Eco denkt Offenheit also mimetisch motiviert, d.h. nicht primär kontrapunktisch, korrigierend, ausgleichend oder kurativ. Vielleicht auch deshalb echappiert ihm die gleichzeitig wirksame, inhaltliche, vielleicht sogar dogmatische Entschiedenheit vieler Kunstwerke. Was, wenn umgekehrt der berühmte Schuh aus der Sache würde? Was, wenn gerade die von Eco konstatierte Unübersichtlichkeit und Unabgeschlossenheit der Moderne im 21. Jahrhundert Verfahren der Schließung und der Entschiedenheit - also Reduktion von Komplexität und Kontingenz - verlangte? Was, wenn - ähnlich wie beim Sport, der seine Popularität daraus bezieht, dass er in einem engen Zeitfenster Gewinner_innen und Verlierer_innen produziert und damit Entscheidbarkeit suggeriert in der zeitgenössischen Kunst Thematiken und Verfahren der Setzung wie der Entschiedenheit attraktiv würden, weil sie es ermöglichten, Antworten zu finden, statt die ohnehin schon quälend offenen Fragen weiter zu massieren? Offenkundig haben sich die zeitgenössischen Kunstwerke von Ecos literarischen Beispielen aus dem Jahr 1962 erheblich entfernt; die interpretative Offenheit tritt zugunsten einer strukturellen Lesart, die Eco im Vorwort zur zweiten Auflage noch einzudämmen versucht, zurück. Mir scheint, dass

14 Ebd., S. 8.

15 Ebd., S. 9. 
Künstler_innen die strukturelle Offenheit des ihre Kunstwerke klug rahmenden Kunstraums strategisch dafür einsetzen, direktere, parteiischere, radikalere Formen der Reflexion anzustoßen.

Um genauer zu verstehen, welche Beziehungen zwischen ,Offenheit ${ }^{\star}$ und ,Entschiedenheit ${ }^{‘}$ von Kunstwerken herrschen, müssen wir näher auf die spezifische Verfasstheit und Wirkungsweise von Kunst eingehen. Dazu lohnt sich - so mein Vorschlag - den Blick auf einige Thesen Niklas Luhmanns zu werfen.

\section{Kunst als kompakte Beziehungsweise:}

weltzugewandt \& körpernah, eigen \& entschieden

In seinem Buch Die Kunst der Gesellschaft von 1995 macht Luhmann, vielleicht ohne es recht zu bemerken, einen interessanten Vorschlag, um die Entschiedenheit und die Offenheit eines Kunstwerks für beide Positionen gewinnbringend miteinander in Beziehung zu setzen, wenn er behauptet, dass sich die Erkenntnis von Kunst einer „im Einzelwerk verdichteten“, gerade nicht diskursiv verfassten „Kompaktkommunikation“ verdanke.16 Diese ist, wie immer beim Systemtheoretiker Luhmann, zwar nicht dialogisch, trotzdem oder sogar eben deshalb ungeheuer beziehungsreich. Ein Kunstwerk wäre dann eine Art non-verbale Form der Behauptung in Gestalt einer raum-zeitlichen ,Wahrnehmungssetzung, deren Anerkennung sich u.a. aufgrund ihrer je eigenen ,Entschiedenheit' nicht automatisch einstellte, sondern offen, kritisch und unwägbar bliebe. Kunst als Kommunikationsweise sei, so Luhmanns Credo, dabei selbst atypisch, da sie unter Umgehung sprachlicher Sinnstiftungsprozeduren durch Wahrnehmung geschehe:

Kunst gewinnt ihre Eigenart daraus, daß sie es ermöglicht, Kommunikation stricto sensu unter Vermeidung von Sprache, also auch unter Vermeidung all der an Sprache hängenden Normalitäten durchzuführen. Ihre Formen werden als Mitteilung verstanden ohne Sprache, ohne Argumentation. ${ }^{17}$

Denkend, so Luhmann weiter, könne man buchstäblich überall sein, d.h. auch in der Vergangenheit, der Zukunft oder in abstrakten Räumen, doch wahrnehmen könne man nur unter dem Einschluss der körperlichen Situiertheit im Hier und Jetzt. ${ }^{18}$

Hier kommt bei Luhmann eine Eigenschaft von Wahrnehmung zur Geltung, die für das Folgende entscheidend sein wird: Wahrnehmung („Fremdreferenz") schließt neben Gegenwärtigkeit („Jetzt-Bewusstsein“) immer Selbstwahrnehmung („Selbstreferenz"), also die eigene Situierung durch und in Gestalt des eigenen Körpers ein. Genau das sei beim Denken ${ }^{19}$ nicht ohne

16 Luhmann, Niklas: Die Kunst der Gesellschaft, Frankfurt a. M. 1995, S. 90.

17 Ebd., S. 39.

18 Ebd., S. 26.

19 Anders als viele analytische Vertreter_innen einer Philosophie des Geistes ist Luhmann umgekehrt nicht davon überzeugt, dass jede Form von Nachdenken auf ähnliche Weise auch Selbstbewusstsein einschließt. 
Weiteres der Fall, so Luhmann weiter. Nur weil der eigene Körper, wenn auch als opaker, notwendig immer schon „mitwahrgenommen" 20 werde, sei Wahrnehmung ,zweiseitig, d.h. weltzugewandt und doch immer auch körpernah, körperbezogen: „Dabei ist der Wahrnehmung die Welt, da sie den eigenen Körper einschließt, komplett, kompakt und undurchdringlich gegeben."21 Ausgehend von dieser weitreichenden Zuspitzung verlagert Luhmann die behauptete Kompaktheit von Weltwahrnehmung im Verlauf von Die Kunst der Gesellschaft nicht ohne Hintersinn scheinbar weg vom wahrnehmenden, menschlichen Körper (der alle Eindrücke in sich versammelt) - in das Kunstwerk hinein. Wahrnehmung und Welt tauschen argumentationslogisch zugunsten des Kunstwerks die Plätze. Durch diesen Schachzug ,erbt das Kunstwerk jedoch die oben beschriebene Zweiseitigkeit, d.h. die körper- wie weltbezogene Janusköpfigkeit der Wahrnehmung, die nun dem Kunstwerk ein- und zugeschrieben wird. Es ,beerbt' die Gegenwärtigkeit des Körpers in potenziell jeder Wahrnehmung und gibt sodann der konkreten Wahrnehmung sowie dem Nachdenken darüber Rätsel auf, und zwar aufgrund der mannigfaltigen Beziehungsweisen, die es stiftet und in die es eingewoben ist. Das Kunstwerk bezieht sich auf diese körpernahe Position qua Ähnlichkeit zurück, wenn auch aus einer gewissen Distanz heraus, die ihr die Künstlichkeit selbst verschafit. Genau diese Distanz verschaffit dem Werk gewisse Freiheiten, ohne deshalb gleich in Beliebigkeit zu verfallen. Statt um Beliebigkeit geht es - so die These - um Entschiedenheit, die oft als opake und nicht immer leicht zu entschlüsselnde Eigenheit wahrgenommen wird. Was sich jedoch vermittelt, ist, wenn nicht Fremdheit qua Künstlichkeit, so doch ein stark idiosynkratisches Moment. Die ,Mitwahrnehmung' des eigenen, die Wahrnehmung überhaupt erst versammelnden Körpers wird blockiert und durch etwas Anderes ersetzt. Ein Kunstwerk evoziert - oder kolportiert - zweifellos etwas Eigenes, das, gerade weil es aufgrund seiner idiosynkratischen Entschiedenheit als unzugänglich erfahren wird, völlig offenlässt, auf welcher Ebene es sich überhaupt abspielt, ob es sich material-körperlich, geistig-konzeptuell oder als ein Hybrid oder als nichts von beidem zu erkennen gibt.

So ,beerbt ${ }^{c}$ dieses Eigene und Entschiedene des Kunstwerks die versammelnde Rolle des (menschlichen) Körpers im Moment der (gewöhnlichen) Wahrnehmung. Nur so ist zu erklären, warum die Wahrnehmung eines Kunstwerks im Ergebnis eine ähnliche Komplettheit, Kompaktheit und Undurchdringlichkeit aufweist wie vormals die Wahrnehmung von ,Welt' bei Luhmann. Das Kunstwerk erscheint so als eine versammelnde und d.h. kompakte Beziehungsweise, weltzugewandt und körpernah, aber eben zugleich eigen und entschieden, welche die von ihm ausgehende ,Kompaktkommunikation mit den Beobachter_innen überhaupt erst begründet. 


\section{IV. ,Kompaktkommunikation durch Wahrnehmung;, weitergelesen mit Gilles Deleuze}

Gilles Deleuze wiederum nennt diese Eigenschaft von Kunstwerken, auf ihre Betrachter_innen weder funktionell noch konzeptuell zu wirken, bereits 1991 perzeptiv. 22

Die Perzepte sind keine Perzeptionen mehr, sie sind unabhängig vom Zustand derer, die sie empfinden; die Affekte sind keine Gefühle oder Affektionen mehr, sie übersteigen die Kräfte derer, die durch sie hindurchgehen. Die Empfindungen, Perzepte und Affekte, sind Wesen, die durch sich selbst gelten und über alles Erleben hinausreichen. Sie sind, so könnte man sagen, in der Abwesenheit des Menschen, weil der Mensch, so wie er im Stein, auf der Leinwand oder im Verlauf der Wörter aufgefaßt wird, selbst eine Zusammensetzung, ein Komplex aus Perzepten und Affekten ist. Das Kunstwerk ist ein Empfindungssein und nichts anderes: es existiert an sich. 23

Das ist keine extended mind theory, sondern eine extended perception theory und als solche auffallend beziehungsreich. Deleuze verbindet mit der Zuordnung des Kunstwerks zu einem „Empfindungsblock“24, ähnlich wie Luhmann vier Jahre später, die Hoffnung, das Chaos der Möglichkeiten einzuhegen, ja, zu reduzieren, nicht obwohl, sondern weil es so komplex ist. ${ }^{25}$ Immer geht es für Deleuze darum, dem Chaos ein bisschen Zeit im ,reinen Zustand' (,un peu de temps à l'état pur“", Proust) abzuringen, ja, diese im und durch das Kunstwerk temporär wahrnehmbar zu machen: „Ist das nicht die Definition des Perzepts überhaupt: die sinnlich unspürbaren Kräfte, die unsere Welt bevölkern, die uns affizieren, uns werden lassen, spürbar zu machen?"26

Zugleich ist aber genau diese - für manche erschreckend esoterisch anmutende - Verselbstständigung und autonome Existenz als „Block von Empfindungen"27 schuld daran, dass das Kunstwerk zum Denken zwingt. Warum? Weil sich im Kunstwerk jede von ihm ausgelöste Empfindung

nur auf sein Material bezieht: Sie ist das Perzept oder der Affekt des Materials selbst, das Lächeln aus Ölfarbe, die Geste aus gebranntem Ton, der Schwung aus Metall, das Gedrungene des romanischen Steins oder das Aufstrebende des gotischen Steins. Und das Material ist in jedem Einzelfall so viel-

22 Deleuze, Gilles / Guattari, Félix: Was ist Philosophie?, übers. von Bernd Schwibs u. Joseph Vogl, Frankfurt a. M. 1996 [zuerst Paris 1991, Qu'est-ce que la philosophie?].

23 Ebd., S. 191f.

24 Ebd., S. 191.

25 „Die Kunst ist nicht das Chaos, wohl aber eine Komposition des Chaos“, ebd., S. 242.

26 Ebd., S. 216.

27 Ebd., S. 196. 
fältig (der Träger der Leinwand, das Wirkende von Pinsel oder Bürste, die Farbe in der Tube), daß es schwierig ist zu sagen, wo die Empfindung tatsächlich beginnt und wo sie aufhört; natürlich gehören die Präparierung der Leinwand, die Spur des Pinselhaars und vieles andere mehr zur Empfindung. ${ }^{28}$

Diesen „Block von Empfindungen“, seine Kompaktheit, Komplettheit und Undurchdringlichkeit, die sich auch als luzide Opazität auffassen lässt, gilt es, von einer auktorialen in eine beziehungsreichere Begriffswahl zu überführen: Da also, wo Deleuze von einem ,Komplex von Perzepten', wo Luhmann von ,Kompaktkommunikation durch Wahrnehmung als Signatur des Kunstwerks spricht, möchte ich den bereits erwähnten Terminus der ,Entschiedenheit ${ }^{\star}$ in die Debatte werfen. Mit ihm wird das Gewicht stärker auf die endliche, eigene und eigensinnige, d.h. idiosynkratische, in seinen Wirkungen nicht selten radikale Konfiguration des Kunstwerks gelegt, ohne deshalb die Möglichkeit einer sich mehr oder minder verselbstständigenden Wahrnehmung (wie bei Luhmann) oder Sensibilität (wie bei Deleuze) aufzugeben. Die Aufmerksamkeit wird auf die besonderen Wirkungen gerichtet, auf die stummen ,Überredungskünste des Kunstwerks, denn Entschiedenheit meint auch umgangssprachlich bekanntlich eine eher schweigsame Verfassung, die nicht zu verbalen Rechtfertigungen neigt.

Zugegeben, ein solcher Vorschlag zur Begrifflichkeit bleibt suggestiv. $\mathrm{Zu}$ rechtfertigen ist er nur, wenn er interessante Wirkweisen von Kunstwerken zutage fördert. Bislang habe ich mit dem Begriff der Entschiedenheit vor allem auf seine künstlerischen oder produktionsästhetischen Gesichtspunkte abgestellt. Im Zentrum stand bislang die Endlichkeit der künstlerischen Mittel und die Konkretion ihrer Gestalt. Es gibt jedoch auch eine rezeptionsästhetische Pointe des Begriffs, um die es im Folgenden gehen soll.

Entschiedenheit umfasst in rezeptionsästhetischer Rücksicht Phänomene wie Rigorosität, Einseitigkeit und Radikalität. Gerade mit Blick auf die bereits entfaltete Doktrin des modernen Kunstdiskurses von der ,Offenheit der Kunstwerke vermute ich, dass Entschiedenheit in diesem Sinne nicht trotz, sondern aufgrund der in ihr mitschwingenden Einseitigkeit ein attraktives Sinnangebot für die Kunstbetrachter_innen bereithält, welche sich in einer komplexen Welt in einer durchaus unbehaglichen Situation wiederfinden.

Entschiedenheit wäre im besonderen Fall der Kunst also etwas, das gerade durch die eigene radikale Andersheit zum Dialog zu einer Neupositionierung zwänge. Die sich anschließende Frage lautet dann: Polarisieren Kunstwerke aufgrund ihrer Entschiedenheit - und wozu könnte das gut sein? Erklärt die Polarisierung bereits die der Kunst eigene Radikalität? Greift für die Kunst und wenn ja, für welche - Helmuth Plessners (mit Blick auf die politischen Jugendbewegungen der 1920er Jahre getätigter) Ausspruch, jede Form des 
,sozialen Radikalismus' sei die „Verunendlichung einer Idee, sei sie rational, sei sie irrational"29? Von welchen Kunstwerken könnte beim Stichwort ,sozialer Radikalismus' sinnvoll die Rede sein? Und sind diese am Ende - überhaupt (noch) Kunst?

\section{V. $245 \mathrm{~m}^{3}$}

Als Beispiel für das Gemeinte möchte ich an Arbeiten von Santiago Sierra erinnern, als deren Signatur Jens Jessen eine „Überbietung der Verkommenheit" ausmachte:

Wo der 39-Jährige auftritt, wird die Öffentlichkeit in helle Aufregung versetzt. Junkies, denen er Streifen ins Haar rasierte, bezahlte er mit Heroin. Arbeitslosen gab er den gesetzlichen Mindestlohn für völlig sinnlose Tätigkeiten. [...] Die Welt, denkt Sierra, ist so verkommen, dass Kunst nur durch Überbietung der Verkommenheit noch etwas bewirken kann. ${ }^{30}$

Anlass für Jessens Artikel aus dem März 2006 war die Arbeit $245 \mathrm{~m}^{3}$ in der Synagoge von Stommeln in der Nähe von Köln. Zum Entsetzen der Besucher_ innen - welche die Synagoge nur einzeln betreten durften, und das auch noch ausgestattet mit einer Atemmaske und begleitet von einem sich sodann auffallend diskret verhaltenden Feuerwehrmann - erzeugten sechs Autos genügend Abgase, um die Synagoge mehr als sechs Jahrzehnte nach dem Holocaust im Wortsinn in eine Gaskammer zu verwandeln. Wobei, das scheint für die heftigen Reaktionen nicht unerheblich, Sierra mit der Wahl seiner Mittel zugleich an eine gängige Selbstmordmethode bankrotter Familienvorstände in amerikanischen Reihenhaussiedlungen mit Doppelgaragen erinnerte. Allerdings könnte die künstlerische Entscheidung für laufende Automotoren auch aus dem pragmatischen Grund der Verdeutlichung durch Versinnlichung gefallen sein: Zyklon B kann man bekanntlich weder sehen noch riechen.

Was es auch sei: Die Existenz der Autos er- und bezeugte einerseits die als banal, fatal, infam oder irrig aufgefasste, im Ergebnis höchst unwillkommene Assoziation eines ,Bilanzselbstmordes'. Genau deshalb entzündete sich an ihr auch polemische Kritik. Alternativ wäre es möglich gewesen, ihre Funktion als Entschiedenheit zu deuten, nämlich als genuin künstlerische Entscheidung, welche auf ästhetische Differenz setzt. Diese Differenz besteht nicht nur zum historischen Holocaust, sondern verweist auch auf das überschießende und vorauseilende Schuldgefühl vieler nach 1945 Geborener, das bedenklich offen, d.h. hier überempfindlich für Instrumentalisierungen aus allen Richtungen ist.

29 Plessner, Helmuth: Grenzen der Gemeinschaft. Eine Kritik des sozialen Radikalismus, Frankfurt a. M. 2002 [zuerst Bonn 1924], S. 17.

30 Jessen, Jens: „Santiago Serra. Warum der Provokationskünstler Gas in eine ehemalige Synagoge leitet“, in: Die Zeit, Nr. 12, 2006, https://www.zeit.de/2006/12/ Santiago_Sierra (09.07.2019). 
Die körperliche Partizipation der Besucher_innen der Synagoge, ihre Involviertheit als Zeug_innen und zugleich als ,Überlebendé eines zweifelhaften Reenactments, ließ jedoch - zugegeben - sehr wenig Raum für ästhetische Distanz, die, laut David Humes Essay „Of Tragedy“, als wesentlich für ästhetischen Genuss anzusehen ist. ${ }^{31} \mathrm{Um}$ diesen betrogen, konnten die Besucher_innen in Stommeln dem giftigen und ohne Atemmaske unvermeidlich todbringenden Kohlenmonoxid mithilfe der lauten Automotoren gewissermaßen dabei ,zuhören', wie es die Synagoge schändete. Der Zentralrat der Juden in Deutschland intervenierte und die Stadt verbot die Aktion schließlich mit Rücksicht auf eine mögliche Verhöhnung der Opfer des Holocaust.

Der Künstler, naturgemäß, fühlte sich missverstanden. Er ließ erklären, seine Arbeit wende sich gerade gegen eine „Banalisierung der Erinnerung an den Holocaust“, indem sie offensiv „das chronische und instrumentalisierte Schuldgefühl" in Deutschland zum Thema mache. ${ }^{32}$ Er hätte auch sagen können: Was habt ihr erwartet, wenn ich Gasmasken vor Betreten einer Synagoge verteile und die echte Feuerwehr bereitsteht? Habt ihr geglaubt, ich erlaube mir einen Spaß? Das hier ist Ernst im Namen einer künstlerischen Wahrheit, die nur ein mögliches Schlaglicht auf eine bekannte historische Tatsache lenkt. Ihr seid ihr nicht gewachsen, wenn ihr euch jetzt zu Opfern eines stillosen Kunstwerkes stilisiert. Eure Freiwilligkeit wollt ihr nicht wahrhaben. Aber genau die führe ich euch vor: Freiwillig habt ihr diese Synagoge betreten. Aus freien Stücken und keineswegs gezwungen, haben eure Eltern und Großeltern tatkräftig dabei geholfen, mehr als sechs Millionen Juden, Homosexuelle, Sinti und Roma sowie politisch Verfolgte in industriellem Maßstab zu vergasen. $\mathrm{Ob}$ aus ideologischer Überzeugung, falschem Pflichtgefühl oder ,Kadavergehorsam ' heraus, ist letztlich egal. Eure Beschwerde ist geschmacklos. Gebraucht endlich euren Verstand. 33

31 „Objects of the greatest terror and distress please in painting, and please more than the most beautiful objects that appear calm and indifferent. The affection, rouzing the mind, excites a large stock of spirit and vehemence; which is all transformed into pleasure by the force of the prevailing movement. It is thus the fiction of tragedy softens the passion, by an infusion of a new feeling, not merely by weakening or diminishing the sorrow. You may by degrees weaken a real sorrow till it totally disappears; yet in none of its gradation will it ever give pleasure; except perhaps, by accident, to a man sunk under lethargic indolence, whom it rouzes from that languid state." Hume, David: „Dissertation III. Of Tragedy“, in: ders.: Four dissertations, London 1757, S. 185-200, hier: S. 192f.

32 Vgl. die Berichte im Tagesspiegel (Berlin) und im Standard (Wien). N. N.: „Synagoge wird zur ,Gaskammer'“, in: Tagesspiegel Online, 12.3.2006, https://www.tagesspiegel.de/ kultur/umstrittene-kunst-synagoge-wird-zur-gaskammer/692546.html (22.07.2019). N. N.: „Santiago Sierra setzt Kunstprojet in Synagoge aus", in: derStandard.at, 21.3.2006, https://www.derstandard.at/story/2374662/santiago-sierra-setzt-kunstprojekt-insynagoge-aus (14.08.2019).

33 Meine Zuspitzung, zu ihrer Rechtfertigung gleich im Fließtext mehr. 
Diese Sätze lege ich hier als Vorschlag Santiago Sierra in den Mund, um das schon erwähnte ,instrumentalisierte Schuldgefühl' konkret an das Moment der Freiwilligkeit der Kunstbetrachtung zurückzubinden. Sierra setzt es nach meinem Dafürhalten so ostentativ ein, dass es als eigenes künstlerisches Ausdrucksmittel durchgeht. Er stellt den Schutzraum der Kunst mittels der Freiwilligkeit, ihn (nicht) zu betreten, planvoll aus. Seine strategische Instrumentalisierung desselben funktioniert wie ein mimetischer Einwand, um zu brüskieren und um zu beschämen, vielleicht auch nur um zu zeigen, wie subtil und offenkundig freiwillig jede Instrumentalisierung (auch die gutgemeinte) vonstatten gehen kann.

In die hitzige Debatte zur Deutungshoheit über das skandalträchtige Werk schaltete sich im Jahr 2006 unter anderem auch Christoph Schlingensief ein. Sein Diktum über die Arbeit seines Künstlerkollegen fiel harsch aus:

Das kann man nicht Kunst nennen. Ein Kunstwerk muss sprechen können, dieses Werk ist in sich schon verstummt. Selbst einem alten „Provokationshasen“ wie mir ist das zu platt. Diese Aktion banalisiert die Aktionen, die Sierra vorher gemacht hat. Sie ist banal, einfach daneben, blöd. ${ }^{34}$

„Ein Kunstwerk muss sprechen können“ - genau das würde Luhmann zugunsten einer kompakteren Form der Kommunikation durch Wahrnehmung bestreiten. Meinte Schlingensief damit, dass es Offenheit im Sinne Ecos brauche - hier: Unschärfe, sodass Plattheit und Plumpheit nicht in pornografischer Direktheit zum plakativen Ziel gelangen? Ist Serras Arbeit $245 \mathrm{~m}^{3}$ also $z u$ entschieden? Ist es deshalb keine ,Kompaktkommunikation durch Wahrnehmung frei nach Luhmann mehr, eben weil sich darin nichts Komplexes ereignet, nichts darin verdichtet?

Oder verhält es sich - anders als Schlingensief behauptet - strukturell ähnlich wie mit dem von Sierra vermauerten spanischen Pavillon 2003 auf der Biennale di Venezia, auch wenn dieser heute, 16 Jahre später, als ,prophetische Intervention ${ }^{35}$ hervorsticht? „Auf der Biennale in Venedig 2003 ließ er [Sierra] den spanischen Pavillon vermauern und den Pass der Besucher kontrollieren - nur Spanier kamen hinein, die anderen sollten sich fühlen wie Asylanten an Europas Grenzen“, kommentiert Jens Jessen. ${ }^{36}$ Geht es nicht hier wie dort um schwer erträgliche, ja desaströse Wahrheiten, die man im

34 Und weiter: „Er soll seine Autos vor den Reichstag stellen und das Gas da reinleiten. Die Politiker könnten sich dann in Gasmasken entrüsten. Mal sehen, was dann los wäre. Die Aktion in der ehemaligen Synagoge von Stommeln ist zu dicht dran.“ Aust, Michael / Schlingensief, Christoph: „Christoph Schlingensief über das Projekt“, in: Kölner Stadt-Anzeiger, 14.3.2006, https:// www.ksta.de/christoph-schlingensief-ueber-das-projekt-13636530 (27.08.2019).

35 Vgl. zu dieser Taktik u. a. Boyd, Andrew / Mitchell, Dave Oswald (Hg.): Beautiful Trouble. A Toolbox for Revolution, New York 2016.

36 Jessen, Jens: „Santiago Serra. Warum der Provokationskünstler Gas in eine ehemalige Synagoge leitet“, in: Die Zeit, Nr. 12, 2006, https://www.zeit.de/ 2006/12/Santiago_Sierra (09.07.2019). 


\section{ART / LIFE ONE YEAR PERFORMANCE}

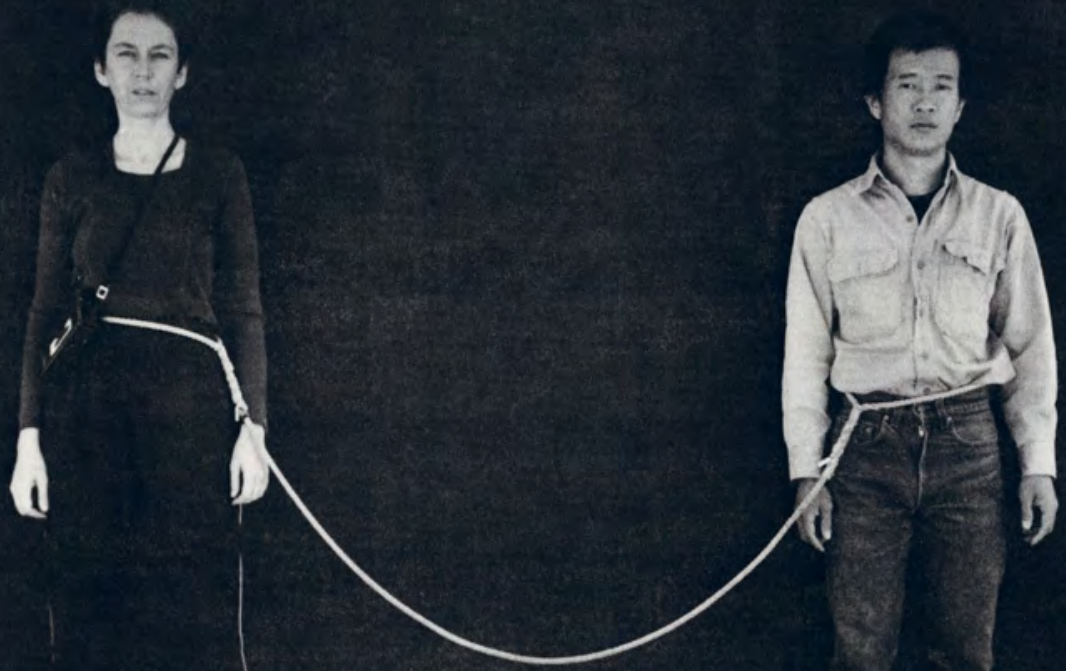

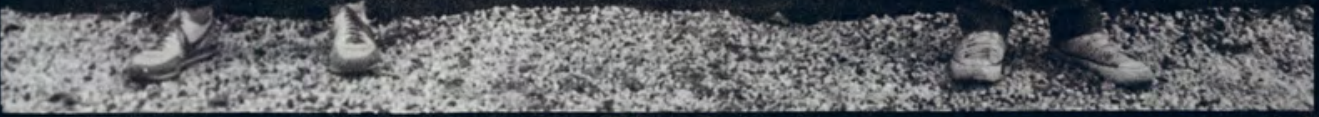

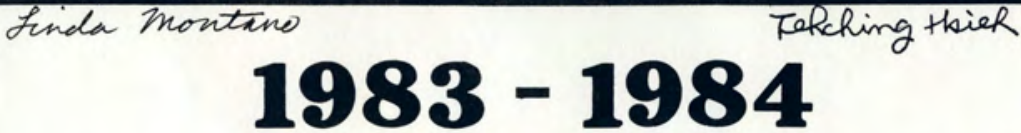

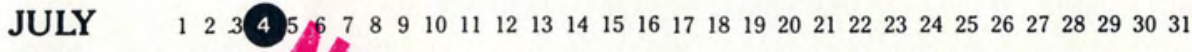
AUG $\quad \begin{array}{llllll}1 & 2 & 3 & 4 & 5 / 6\end{array}$

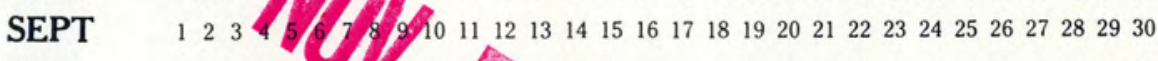
OCT $\quad \begin{array}{lllllll}1 & 2 & 3 & 4 & 5 & 6 & 7\end{array} \quad 9101213141516171819202122232425262728293031$

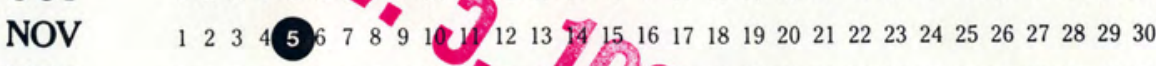

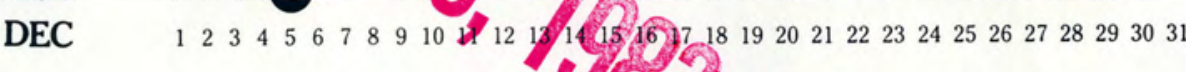
020

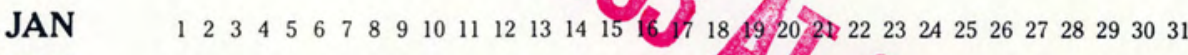

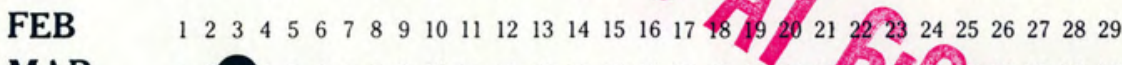

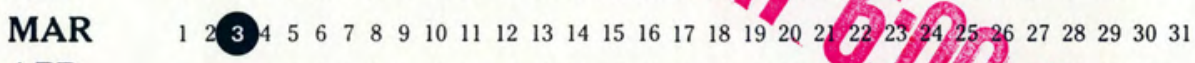

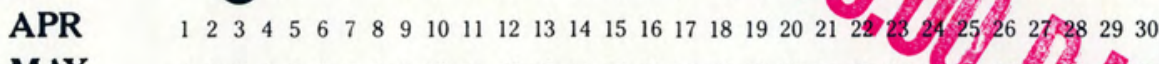

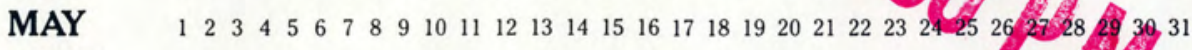

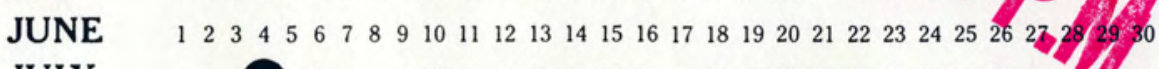

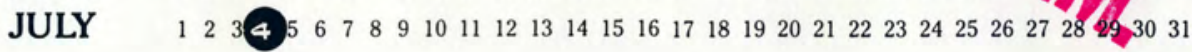


Schutz eines Kunstwerks gerade nicht erwartet? Wohnt Arbeiten wie diesen nicht eine Form der Publikumsbeschimpfung oder -beleidigung als Mittel der Aufrüttelung inne, welche die Grenze zwischen Kunst und Leben absichtsvoll verletzt, indem es die schützende ästhetische Distanz von sich aus einseitig aufkündigt? Wer sich über die Ausweiskontrolle am Vordereingang der spanischen Pavillons in den venezianischen Giardini aufregt, um dann mit einem spanischen Pass doch den Hintereingang zu nehmen, was erwartet der_diejenige ernstlich vom Innern? Darin befindet sich nichts von Belang und schon gar kein Kunstwerk - nur andere spanische Passbesitzer_innen, die meinten, von ihrem Eintrittsprivileg Gebrauch machen zu müssen, nur um sich vom Künstler Sierra mithilfe ihrer eigenen Prätentionen düpieren und vorführen zu lassen. ${ }^{37}$

VI. Was zum Denken zwingt:

Beziehungsweisen zwischen der Offenheit und der Entschiedenheit des Kunstwerks

Am Anfang des Denkens steht der Einbruch, die Gewalt, der Feind [...], die Kontingenz einer Begegnung mit dem, was zum Denken nötigt, um die absolute Notwendigkeit eines Denkaktes, einer Leidenschaft zum Denken aufzureizen und anzustacheln. [...] Es gibt etwas in der Welt, das zum Denken nötigt. Dieses Etwas ist Gegenstand einer fundamentalen Begegnung, und nicht einer Rekognition. ${ }^{38}$

Gilles Deleuze möchte mit dem ,Idyllenporträt' Schluss machen, das das Denken gerne von sich selber zeichnet: nämlich ein Denken zu sein, das ganz bei sich ist und das mit jedem neuen Gedanken mehr an Gewissheit gewinnt usf. Deleuze will bereits in Differenz und Wiederholung (1968) dieses sogenannte ,Bild des Denkens', das in repräsentationalen und somit identifizierenden Begriffen oder in dialektischen Prozeduren zu Hause war, korrigieren und durch ein weniger idyllisches Bild ersetzen, das vom Einbruch des Nichtvorhergesehenen, ja Unkontrollierbaren, Nichteingehegten kündet und eben zum Denken - durchaus unfreiwillig, durchaus gewaltsam - nötigt, sich selbst eher als ,Beute' von etwas denn als ,Jäger' einer absoluten Wahrheit begreifend.

37 Es gibt übrigens eine nicht unähnliche, ähnlich ernüchternde Arbeit mit dem Titel 7-8:30 PM 05.31.2007 von Gregor Schneider, die - mithilfe von bezahlten Kompars_innen neben ahnungslosen Zuschauer_innen - ein stundenlanges SchlangeStehen vor dem Depot der Staatsoper Berlin vorsah, nur um im Eilschritt durch das leere Innere geschleust respektive gescheucht zu werden. Wer danach verärgert ins Freie trat, lief in eine Kamerafalle, die alle Emotionen der Enttäuschung auch noch für die Ewigkeit festhielt, wurden doch zumindest einige der Fotos anschließend von Schneider in einem Buch veröffentlicht, siehe Schneider, Gregor: 19-20:30 Uhr 31.05.2007. 7-8:30 PM 05.31.2007, hg. von Staatsoper unter den Linden und ThyssenBornemisza Art Contemporary, Köln 2007.

38 Deleuze, Gilles: Differenz und Wiederholung, übers. von Joseph Vogl, München 1992 [zuerst Paris 1968, Différence et Repetition], S. 181f. 
Erkenntnis stellt sich vor diesem Hintergrund beim Betrachten von Kunstwerken nicht zwangsläufig und nicht regelerzeugt ein. Erkenntnis vor Kunstwerken geschieht oft überfallsartig, d.h., sie besitzt im Unterschied zum langsam wachsenden Verständnis neben der Verblüffung durchaus so etwas wie einen ,Offenbarungscharakter. Eine solche Erkenntnis schmerzt, sticht, tut weh, denn sie ist mit der Annahme oder Akzeptanz einer unangenehmen oder gar anstößigen Wahrheit verbunden. Entschiedenheit begründet und ergründet sich nicht, sondern arbeitet mit Setzungen, die ihre eigene Autorität entfalten. Besonders ausgeprägt sind sie in der Installation \& Performance Art, wo oft genaue Handlungsweisen vorgegeben werden, ohne dass ihr - oft auf den ersten Blick obszöner oder anstößiger - Sinn deutlich wird.

So beschließen die vorher einander nur flüchtig bekannten PerformanceKünstler_innen Linda Montano und Tehching Hsieh im Sommer 1983, für ein Jahr lang verbunden zu sein durch ein acht Fuß, also knapp zweieinhalb Meter langes, fingerdickes Seil, das beide um die Hüften gebunden tragen, Tag und Nacht. ${ }^{39}$ Alles ist möglich, sie können gemeinsam überall hingehen, Menschen treffen, lesen, essen, schreiben, schlafen - sowie sich unterhalten, diskutieren, streiten, wohin sie gehen, wen sie treffen usf. Es gibt nur ein Gebot, ein Verbot zu beachten, das vermutlich - ähnlich wie im Fall von Lots Frau oder bei Orpheus, der sich nicht nach Eurydike umdrehen darf - genau deshalb den unwiderstehlichen Reiz der Übertretung auslöst: Beide dürfen sich in diesem Jahr nicht berühren. Es macht die Versuchsanleitung dieser endurance art zu einer wirklichen Tortur, oder, wahlweise, zu einer heilsamen Kur, da sie beiden ein Minimum an ,Privatheit' erlaubt, allerdings um den Preis, sie zugleich jeder allzu menschlichen Regung zu berauben, nämlich beieinander Schutz und Hilfe zu suchen: Sich nicht zu berühren, während man (freiwillig) ein Jahr lang nur zweieinhalb Meter voneinander entfernt, aneinander gefesselt, ja gekettet ist, ist das nicht - eine übermenschliche Forderung? Schaffen die beiden das? Und wenn ja, was macht dieses Jahr aus beiden? Die Performance heißt schlicht: Rope Piece. Die Versuchsanleitung selbst ist voller Entschiedenheit. Es gibt keine Rechtfertigung, keine Erklärung für das ausgesprochene, schriftlich fixierte ,Gebot/Verbot' der Nicht-Berührung. Es ist auch nicht mit Sicherheit bekannt, ob das Gebot/Verbot eingehalten oder übertreten wurde. Bekannt ist nur, was Tehching Hsieh über seine Performance-Partnerin später mitteilte:

I think Linda is the most honest person I've known in my life and I feel very comfortable to talk - to share my personality with her. That's enough. I feel that's pretty good. We had

39 Hsieh hält sich zu der Zeit illegal in den USA auf und beginnt mit einer Reihe von Performances, die immer genau ein Jahr dauern, wie das Cage Piece (1978/79), Time Clock Piece (1980/81) und das Outdoor Piece (1982/83) und die u. a., wie die Titel schon sagen, das Leben im Freien oder in einem Käfig einschließen. Nach Rope Piece folgen noch No Art Piece (1985/86) sowie Thirteen Year Plan (1986-1999). Vgl. mehr hierzu in: Heathfield, Adrian / Hsieh, Tehching: Out of Now. The Lifeworks of Tehching Hsieh, London, Cambridge (MA) 2009. 
a lot of fights and I don't feel that is negative. Anybody who was tied this way, even if they were a nice couple, I'm sure they would fight, too. This piece is about being like an animal, naked. We cannot hide our negative sides. We cannot be shy. It's more than just honesty - we show our weakness. ${ }^{40}$

„Was wir Kunst nannten, beginnt erst zwei Meter vom Körper entfernt", heißt es bei Walter Benjamin. ${ }^{41}$ Wir müssen uns Linda Montano und Tehching Hsieh als seine Leser_innen vorstellen, denn diese Performance wagt die Probe auf Benjamins Diktum.

\section{Acht Thesen zum Schluss}

1. Kunstwerke vermitteln komplexe Haltungen gegenüber uralten Problemen und kulturellen Befindlichkeiten. Sie sind gebrochene Spiegel ihrer Zeit und Kultur, denn sie sind Figurationen eines radikal anderen Wissens und Erkennens.

2. Kunstwerke funktionieren wie "sensibilia“ (Deleuze) in den Dingen; sie zeigen mit sinnlichen Mitteln dem sie umkreisenden Denken dessen eigene Aporien auf. Sie sind auch deshalb eine Zumutung und Provokation, weil sie so auf die Grenzen des räsonierenden, kritischen wie dialogischen Denkens verweisen.

3. Aufgrund der ihnen eigentümlichen „Kompakt-Wahrnehmung“ (Luhmann) sind Kunstwerke ein verdichtetes Kommunikationsangebot, das man gewissermaßen nicht ablehnen kann; dabei sind sie selbst nicht dialogisch, nicht argumentativ aufgebaut. Gerade ihre Stummheit reizt und fordert zahllose und streitbare Interpretationen heraus.

4. Ins Zentrum rückt damit die besondere Entschiedenheit von Kunstwerken, womit die bekannte Rede von ihrer Geschlossenheit in Ergänzung zu ihrer von Eco konstatierten multilateralen Offenheit epistemisch gewendet wird.

5. Jedes Werk ist durchdrungen von einer Unzahl getroffener oder auch vermiedener künstlerischer Entscheidungen, die auch den Zufall strategisch einschließen oder ausschließen können. Entschiedenheit meint - produktionsästhetisch aufgefasst - einfach die endliche Wahl der künstlerischen Mittel, Materialien und Formate, darin eingeschlossen ihre Aufführungsbedingungen und diskursiven Rahmungen.

40 Tehching Hsieh, zit. n. Sara Araujo: „How Being Tied To Another Person For One Year Taught This Artist About Freedom And Humanity", in: Cultura Colectiva, 16.1.2018, https://culturacolectiva.com/art/tehching-hsieh-oneyear-performance (22.07.2019).

41 Benjamin, Walter: „Traumkitsch“ [zuerst 1927], in: ders.: Gesammelte Schriften, Bd. II.2, hg. von Rolf Tiedemann u. Hermann Schweppenhäuser, Frankfurt a. M. 1991, S. 620-622, hier: S. 622.

42 Dieter Mersch nennt diesen Anstoß, etwas Singuläres zu denken, das „Reservoir der Kunst“, ihre „negative Potenz", ihre „Idiosynkrasie“, vgl. Mersch, Dieter: Epistemologien des Ästhetischen, Zürich/Berlin 2015. Roland Barthes würde von ihrem „Punctum“ sprechen. 
6. In der Installations- und in der Performance-Kunst ist diese Entschiedenheit besonders ausgeprägt. An ihrem Beispiel erklärt sich, warum sie - rezeptionsästhetisch gewendet - ihren Betrachter_innen andere, direktere, lokalere und parteiischere Formen der Reflexion abverlangt, als dies gewöhnlich philosophische Diskurse tun. ${ }^{42}$

7. Diese konkrete Entschiedenheit ist mindestens so attraktiv wie ihre strukturelle Offenheit, zumal in komplizierten Zeiten, in denen radikale und populistische Sinnangebote Hochkonjunktur haben.

8. Die besondere Bezüglichkeit der Kunst besteht gerade darin, Entschiedenheit und Offenheit zusammen, nicht als Gegensätze denken zu müssen. Gerade dieses Junktim aus Entschiedenheit/Geschlossenheit und Offenheit/Unabschließbarkeit verbindet ihre Betrachter_innen ähnlich wie die endurance artists Linda Montano und Tehching Hsieh über einige Armlängen Distanz hinweg. Es setzt die Betrachter_innen unter gedanklichen Zugzwang, der jedoch nicht tyrannisch, sondern notwendig und gerechtfertigt wirkt, da sein exemplarischer Wert nicht infrage steht.

\section{Ghassan Salhab und Michaela Ott im Gespräch \\ Film als \\ Gegenverwirklichung}

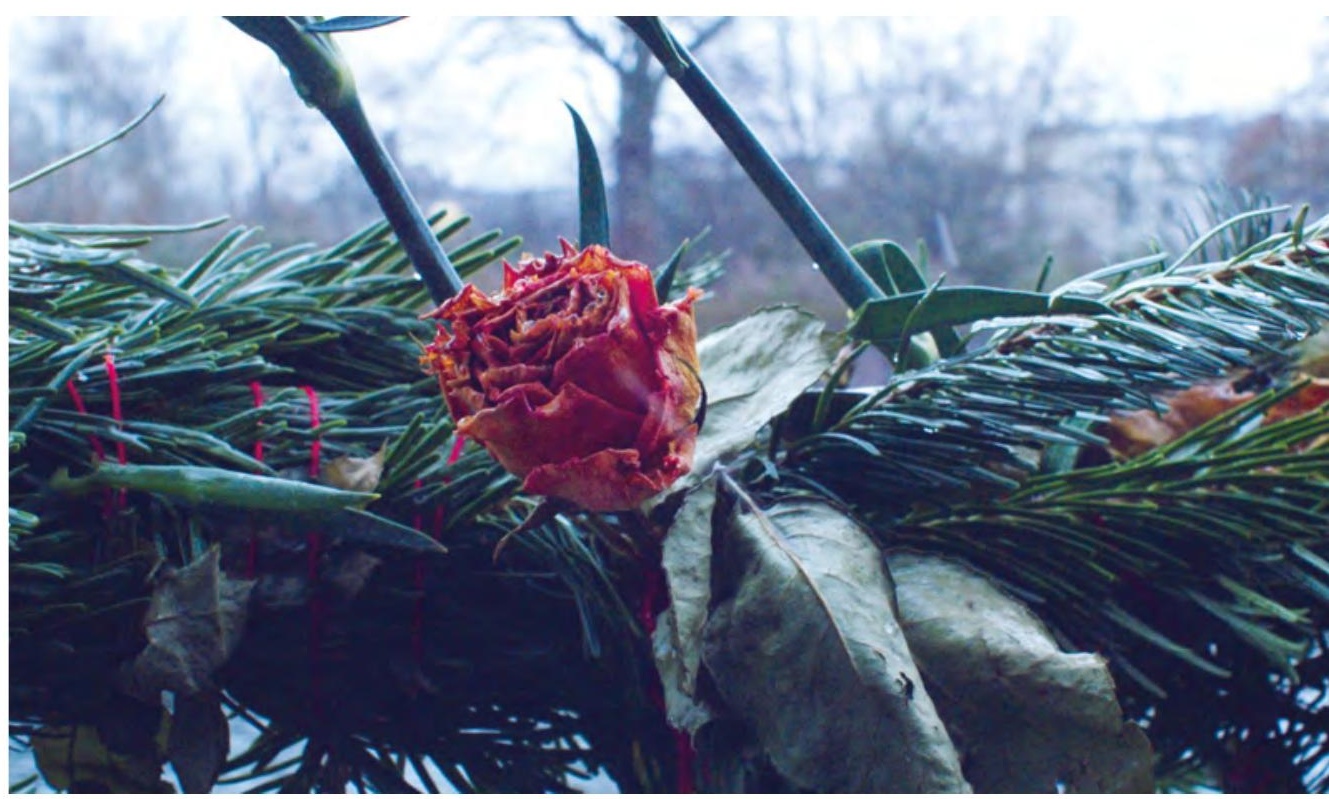

Abb. 1-9 Ghassan Salhab: Une rose ouverte / Warda, 2019, Filmstills 